\title{
Surface edge state and half-quantized Hall conductance in topological insulators
}

\author{
Rui-Lin Chu, ${ }^{1}$ Junren Shi, ${ }^{2}$ and Shun-Qing Shen ${ }^{1}$ \\ ${ }^{1}$ Department of Physics and Center of Computational and Theoretical Physics, The University of Hong Kong, Pokfulam Road, Hong Kong \\ ${ }^{2}$ International Center for Quantum Materials, Peking University, Beijing 100871, China \\ (Received 8 March 2011; revised manuscript received 25 June 2011; published 23 August 2011)
}

\begin{abstract}
We study the surface local density of states and the transport properties of a three-dimensional (3D) topological insulator (TI) in the presence of a uniform spin-splitting Zeeman field. We find chiral edge states exist on the gapped surfaces of the 3D TI, which can be considered as interface states between domains of massive and massless Dirac fermions. Effectively these states are the result of splitting of a perfect interface conducting channel. This picture is confirmed by the Landauer-Büttiker calculations in four-terminal Hall bars made of 3D TIs. It is demonstrated that the difference between the clockwise and counterclockwise transmission coefficients of the two neighboring terminals is approximately one-half, which suggests that the half-quantized Hall conductance can be manifested in an appropriate experimental setup. We also predict that the quantized anomalous Hall effect exists in thin films of TIs where such effective Zeeman felds are present.
\end{abstract}

DOI: 10.1103/PhysRevB.84.085312

PACS number(s): 73.20.-r, 73.43.-f

\section{INTRODUCTION}

Topological insulators are insulating in the bulk, but their metallic surface states can be considered as massless fermions whose dispersion form an odd number of Dirac cones. ${ }^{1-3}$ The band structure and the quantum spin texture of these surfaces states have been well established theoretically and experimentally. ${ }^{4-9}$ In the presence of a spin-splitting Zeeman field, which could be induced by magnetically doping the samples or putting the samples in the proximity of ferromagnetic materials, the Dirac fermions will gain a finite mass and the spectrum will open a gap. ${ }^{10,11}$ When the Fermi surface is located within this energy gap, it was proposed that the Hall conductance of the surface states will be one-half of the conductance quantum $e^{2} / h \cdot{ }^{12-14}$ Based on this, Qi et al. proposed the unconventional magneto-electric effect, which is regarded as one of the characteristic features of the topological insulators. ${ }^{14-16}$

In the usual quantum Hall systems, the current-carrying chiral edge states are responsible for the integer quantized Hall conductance measured in the transport experiments. ${ }^{17,18}$ It is not immediately clear if similar chiral edge states are responsible for the half-quantized Hall conductance in topological insulators, and how the quantized nature of the edge conducting channels can be reconciled with the prediction of the half quantization of the Hall conductance. ${ }^{12-14}$

To address these issues, we consider a topological insulator subjected to a uniform spin-splitting Zeeman field. Instead of using a two-dimensional (2D) effective model (i.e., the massive Dirac Hamiltonian), which has been commonly used in various literatures, we use a real three-dimensional (3D) effective Hamiltonian that is proposed for 3D TIs. ${ }^{7,19}$ The reason for doing this is that a 3D TI has different surfaces, (e.g, six facets for a cubic TI), and electrons on each surface can be considered as 2D Dirac fermions. As we will show below, in a uniform spin-splitting Zeeman field, electrons on the different facets gain different properties. And thus a 2D effective model cannot describe the physics adequately since it is a $3 \mathrm{D}$ problem. ${ }^{20}$

Taking a cubic TI as an example, which is shown in Fig. 1, in a uniform spin-splitting Zeeman field, the top and bottom surface Dirac cones opens an energy gap but the side surface Dirac cones remain intact. Effectively the system becomes two insulating domains of massive Dirac fermion with opposite masses separated by massless Dirac fermion in the middle. We show that chiral edge modes will form at the interfaces of the massive and massless domains, resulting in chiral surface edge states on the top and bottom surfaces. We show that these surface edge states can be viewed as the result of the splitting of a perfect interface conducting channel in two dimensions. Thus each of these surface edge states carries one-half of the conductance quantum $\left(e^{2} / h\right)$. We verify the above physical picture by calculating the local density of states(LDOS) on the TI surfaces. As predicted by this picture, we find chiral current densities on the surface edges. We also do Landauer-Büttiker calculations on a 3D device made of TIs. The result shows that a quantized anomalous Hall conductance of $e^{2} / h$ exists in the thin TI samples but reduces to $e^{2} / 2 h$ in the thick sample limit.

\section{EFFECTIVE MODEL AND PHYSICAL PICTURE}

\section{A. Effective Hamiltonian}

To illustrate the basic physics, we consider a 3D TI of cubic shape. A Zeeman field is applied along the $Z$ direction, as shown in Fig. 1. Because the bulk of the system is insulating, it is effectively a closed 2D surface with six facets. ${ }^{20}$ The effective Hamiltonian of the Dirac fermions for the surface state can be written as ${ }^{19,21}$

$$
H_{\mathrm{eff}}(\boldsymbol{k})=v \hbar(\boldsymbol{k} \times \boldsymbol{\sigma}) \cdot \boldsymbol{n}-g_{\|} \mu_{B} h_{\|} \sigma_{\|}-g_{\perp} \mu_{B} \boldsymbol{h}_{\perp} \cdot \boldsymbol{\sigma}_{\perp},
$$

where $\boldsymbol{n}$ denotes the normal vector of the surface, $\sigma \equiv$ $\left\{\sigma_{x}, \sigma_{y}, \sigma_{z}\right\}$ are the Pauli matrices, $h_{\|}\left(\sigma_{\|}\right)$and $\boldsymbol{h}_{\perp}\left(\sigma_{\perp}\right)$ are the Zeeman field (Pauli matrix) components parallel and perpendicular to the normal vector, respectively, and $g_{\|}$and $g_{\perp}$ are the corresponding spin $g$ factor. It is noted that the surface state has anisotropic spin $g$ factor due to strong spin-orbit coupling of the bulk: $g_{\|}$is the same as the $g$ factor of the bulk material while $g_{\perp}$ is renormalized by bulk band parameters and is usually strongly suppressed. ${ }^{19,21}$ The different facets have different effective Hamiltonians respective to the different normal vectors $\boldsymbol{n}$. For the top and 

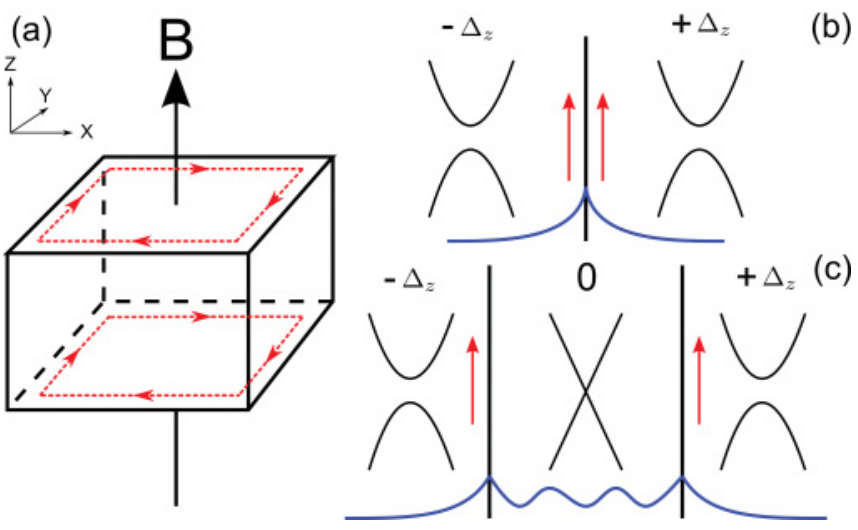

FIG. 1. (Color online) (a) Schematic of a 3D TI in a weak Zeeman field, and the formation of chiral current on the top and bottom surface boundaries. (b) A chiral edge state will form around the domain wall between the 2D Dirac fermions with positive and negative masses, and the wave function is illustrated. The arrow indicates the flow of edge current. (c) When the sharp domain wall evolves to finitewidth metallic band, the edge mode is effectively split to two halves concentrated around the two boundaries.

the bottom facets, the effective Hamiltonian can be written as $H_{\text {eff }}= \pm v \hbar\left(k_{x} \sigma_{y}-k_{y} \sigma_{x}\right)+\Delta_{z} \sigma_{z}$, where $+/-$ is for the top/bottom surfaces, and $\Delta_{z} \equiv-g_{\|} \mu_{B} h$. The spectrum will open a gap on these facets, and the Dirac fermions gain mass $\pm \Delta_{z} / v^{2}$. On the other hand, the effective Hamiltonians for the side facets can be written as $H_{\text {eff }}=v \hbar\left[\left(k_{x}+\Delta k\right) \sigma_{z}-k_{z} \sigma_{x}\right]$, where $\Delta k \equiv g_{\perp} \mu_{B} h$. In this case, the Zeeman field simply shifts the Dirac point from $\left(k_{x}=0, k_{z}=0\right)$ to $(-\Delta k, 0)$.

\section{B. Interface states of Dirac fermions with different masses}

When the Fermi surface is located in the gap of the top and bottom surface, the system becomes effectively two insulating domains (top and bottom surfaces) sandwiching a conducting belt of massless Dirac fermions (side surfaces).

The Dirac fermions on the top and bottom facets have opposite masses because the normal vectors for the two surfaces are in the opposite directions. If we consider an extreme case where the sample has vanishing thickness along the $Z$ direction but ignore the coupling between the top and bottom surfaces, the system is locally equivalent to a domain-wall structure across which the Dirac fermion mass changes sign from positive to negative, as shown in Fig. 1(b). The change of the band topology necessitates the close of the energy gap around the domain wall, giving rise to the gapless interface mode. By directly solving the wave equation and matching the wave functions at the boundary, it is easy to obtain the wave function of the interface mode in the form $\Psi(x, y)=\sqrt{\Delta_{z} / 4 \pi \hbar v}(1,1)^{T} \exp \left[-\left(\Delta_{z} / \hbar v\right)|x|+\mathrm{i} k_{y} y\right]$. Its dispersion is linear in the momentum: $E=-v \hbar k_{y}$. This interface mode forms an ideal one-dimensional (1D) chiral edge channel carrying a quantized conductance $e^{2} / h$.

When the thickness of the sample along the $Z$ direction is finite, the two insulating domains are separated by a finite region of massless Dirac fermion, equivalent to the configuration shown in Fig. 1(c). In this case, the electrons in the metallic region will form subbands due to the confinement by the insulating domains. The normal subband has the dispersion $E_{n}= \pm \hbar v \sqrt{k_{y}^{2}+k_{n}^{2}}$, where $k_{n} \neq 0$ is one of the discrete momentum of the eigenmodes along the confinement direction. Beside these, there is a chiral solution with the dispersion $E=-\hbar v k_{y}$, corresponding to $k_{n}=0$ but with only the left-going mode. The chirality is also shown in the penetration amplitude of the normal subband wave function into the insulating domains: The penetration amplitude minimizes when $E \sim-\hbar v k_{y}$, and increases away from it. All subbands crossing the Fermi surface will contribute to the transport properties, and form an effective chiral edge state as a result of collective combination. The chirality will give rise to a finite Hall conductance.

\section{INDICATION OF CHIRAL SURFACE EDGE STATES FROM SURFACE LDOS}

To carry out numerical studies on these chiral states, we start with a tight-binding Hamiltonian on a cubic lattice for an isotropic 3D topological insulator:

$$
\mathcal{H}=\sum_{i} c_{i}^{\dagger} \mathcal{M}_{0} c_{i}+\sum_{i, \alpha=x, y, x}\left(c_{i}^{\dagger} \mathcal{T}_{\alpha} c_{i+\alpha}+c_{i+\alpha}^{\dagger} \mathcal{T}_{\alpha}^{\dagger} c_{i}\right),
$$

where

$$
\begin{aligned}
\mathcal{T}_{\alpha} & =B \sigma_{z} \otimes \sigma_{0}-i \frac{A}{2} \sigma_{x} \otimes \sigma_{\alpha}, \\
M_{0} & =(M-6 B) \sigma_{z} \otimes \sigma_{0}+\Delta_{z} \sigma_{0} \otimes \sigma_{z} .
\end{aligned}
$$

The lattice space is taken to be unity. The spin-splitting Zeeman field is represented as $\Delta_{z} \sigma_{0} \otimes \sigma_{z}$, which means that it is present throughout the whole bulk. Near the $\boldsymbol{k}=0$ point in the momentum space (i.e., the low energy regime), this model is reduced to a Dirac-like model, which can be derived from either the theory of invariants, ${ }^{7}$ or the eight-band extended Kane model near the region where the $\Gamma_{6}^{-}$and $\Gamma_{8}^{+}$bands invert. The Dirac model for a topological insulator has been discussed by several authors. ${ }^{14,23}$ It was shown that the model yields the effective surface Hamiltonian Eq. (1) with $v=A$. $^{19,21}$

The existence of the gapless surface states can be demonstrated by calculating the surface LDOS. Because the surface states reside dominantly on the surfaces, they project much larger LDOS on the surfaces than the bulk states. ${ }^{22}$ In Fig. 2

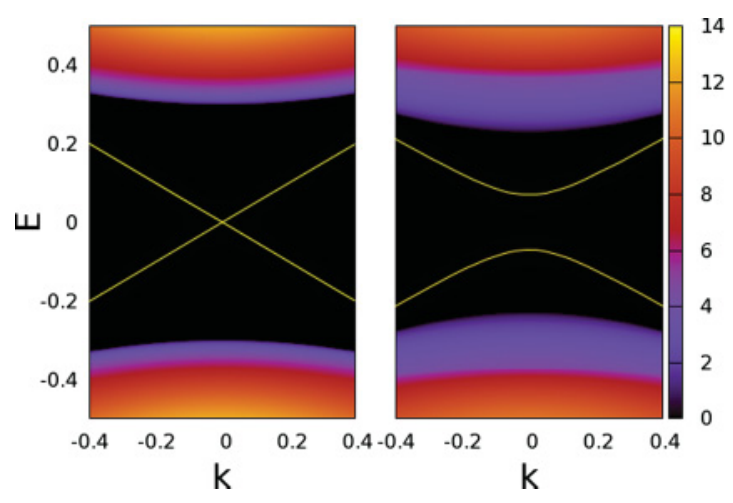

FIG. 2. (Color online) Local density of state on an infinite XY surface of a semi-infinite 3D system. (Left) Gapless single Dirac cone of the surface state; (right) gap opening by application of a Zeeman splitting term. The model parameters are $A=0.5, B=0.25$, $M=0.3$, and $\Delta_{z}=0.07$. 
we plot the LDOS $\rho\left(k_{x}, k_{y}\right)$ of a top surface that is infinite in the $X$ and $Y$ dimension. The $Z$ dimension is semi-infinite with $z \in[0,-\infty)$. In this case, $k_{x}$ and $k_{y}$ are good quantum numbers. A quick recursive approach is employed to calculate the Green's function for a few top layers. ${ }^{24}$ LDOS at the top surface is given by

$$
\rho_{i}\left(k_{x}, k_{y}\right)=-\frac{1}{\pi} \operatorname{Tr}\left[\operatorname{Im} G_{i i}\left(k_{x}, k_{y}\right)\right]
$$

where $G_{i i}$ is the retarded Green's function for the $i$ th layer from the top. In the absence of the Zeeman field, the gapless linear Dirac cone can be observed in the bulk band gap (Fig. 2, left panel). The spatial dependence of LDOS along the $Z$ axis shows that the states in the Dirac cone reside dominantly near the top surface, which is the signature of the surface states in 3D topological insulators, and is also consistent with the analytical solutions. ${ }^{19,22}$ In the presence of a Zeeman field, the LDOS shows that a gap of magnitude $2 \Delta_{z}$ is opened in the surface state Dirac cone as expected (Fig. 2, right panel). Similarly, a gap of the same magnitude is also opened in the Dirac cone of the bottom surface. But effectively when we transform the bottom surface onto the same surface with the top, the two Dirac cones have opposite gap signs, as is mentioned in previous sections. However, the Dirac cones on the side surfaces remain gapless in this Zeeman field.

As is expected from the effective model, we expect chiral states exist on the top surface edges in the Zeeman gap. To check this prediction and see how these states distribute, we study a geometry that is finite in the $Y$ dimension, infinite in the $X$ dimension, and semi-infinite in the $Z$ dimension, as illustrated in the upper panel of Fig. 3. In this case only $k_{x}$ is
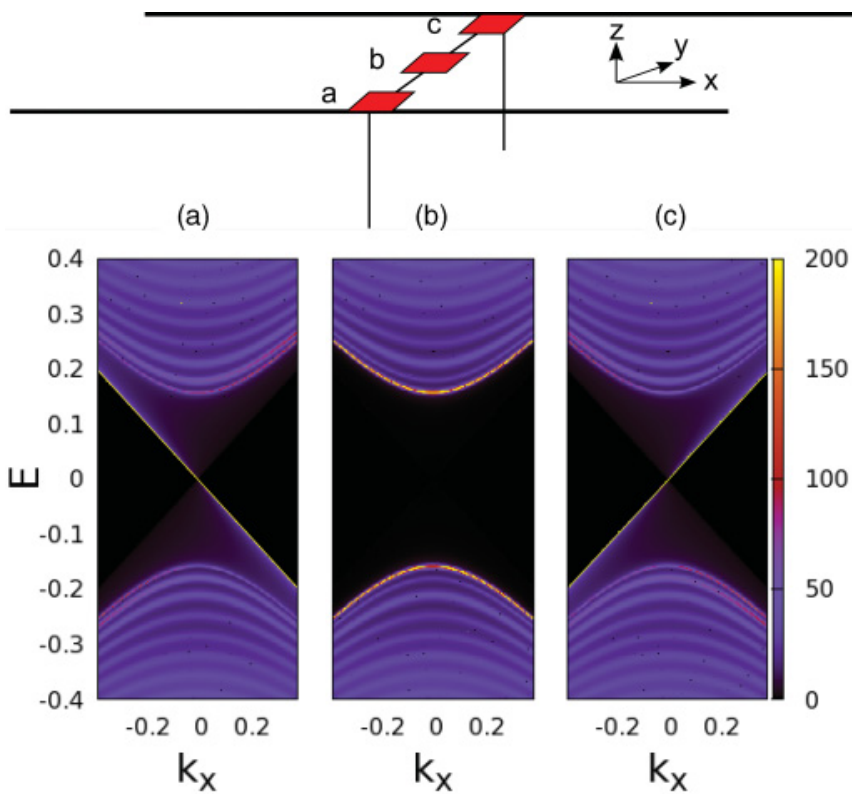

FIG. 3. (Color online) LDOS on the top surface of a structure that is infinite in the $X$ direction, finite in the $Y$ direction, and semi-infinite in the $Z$ direction. Sampling is taken correspondingly in the (a), (b), and (c) regions as illustrated in the upper panel. $\Delta_{z}=0.15, M=0.4$, and $L_{y}=30 a ; a$ is the lattice space. a good quantum number. The LDOS is

$$
\rho\left(y, k_{x}\right)=-\frac{1}{\pi} \operatorname{Tr}\left[\operatorname{Im} G_{00}\left(y, k_{x}\right)\right] .
$$

We can do an average on the LDOS at three typical regions (a)-(c) along the $Y$ direction as shown in the lower panel of Fig. 3. It is found that nonvanishing density of states emerges in the gap at the regions (a) and (c) which are close to the edges of the top surface. But in the region (b) there is no state emerging in the Zeeman gap since the top surface is supposed to be insulating. This means that the states emerging in regions (a) and (c) are interface states and reside on the surface edges. The chiral nature of these in-gap states can be directly observed on the plot of LDOS: The LDOS at the edge (a) maximizes at $E=-v k_{x}$, while the LDOS at the edge (c) maximizes at $E=v k_{x}$. This means that the states on the edge (a) are mostly left going while those on edge (c) are mostly right going. As a result, these states form chiral surface edge states. Compared with conventional chiral edge states in the quantum Hall systems, these chiral surface edge states are not single energy modes, but they are also expected to carry a Hall conductance, whose value will be studied in the following sections.

\section{SURFACE-EDGE CHIRAL CURRENT DENSITIES}

The nonsymmetric profile from the LDOS plot in Fig. 3 implies that a chiral current flows on the top surface edges. We can calculate the current density profile at the Fermi surface $E_{f}$ along the $Y$ direction using

$$
\left\langle J_{x}(y)\right\rangle=i e \int_{k_{x}} \operatorname{Tr}\left[v_{x}\left(r, k_{x}\right) G^{<}\left(r, k_{x}\right)\right],
$$

where $v_{x}=\partial H / \partial k_{x}$ is the velocity operator and $E_{f}$ is in the Zeeman splitting gap. In the equilibrium condition we have $G^{<}=f\left(E_{f}\right)\left(G^{a}-G^{r}\right)$, where $G^{a}$ and $G^{r}$ are the advanced and retarded Green's function and $f\left(E_{f}\right)$ is the Fermi-Dirac distribution function and we assume zero temperature. In Fig. 4(a) the current density distribution along the $Y$ direction
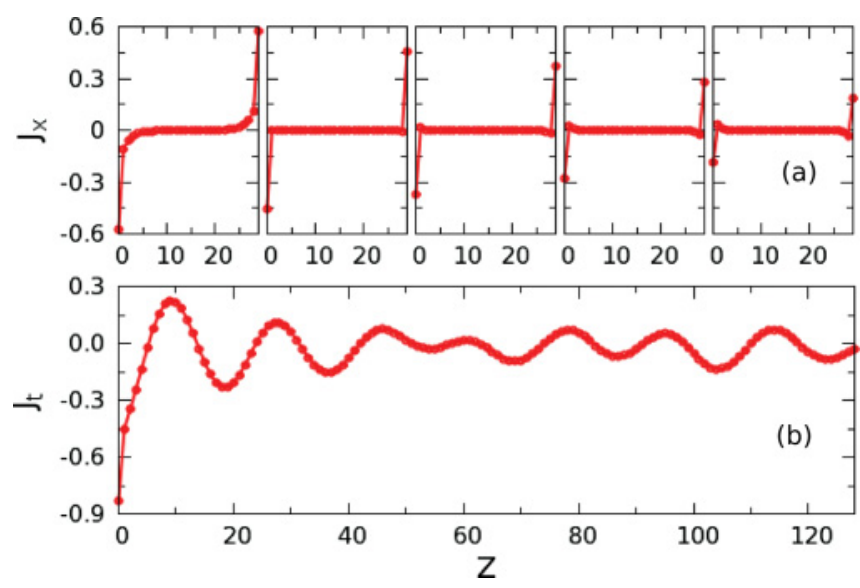

FIG. 4. (Color online) (a) Local current density $J_{x}$ distribution along the width of Y $\left(L_{y}=30 a\right)$ for the top five layers (the leftmost is the first); (b) Total chiral current density $J_{t}$ as a function of layer depth $Z$. $\Delta_{z}=0.15, M=0.4, E_{f}=0.075$. 
for the top five layers is shown. It can be seen that the current is localized dominantly near the edges and is of opposite sign on the opposite edges of the top surface, which implies the chiral nature of the current. To see how the chiral current is concentrated on the surface edges, we sum up the current density for each layer over half the width of the $Y$ direction, $J_{t}=\sum_{0}^{L_{y} / 2} J_{x}(y)$, and observe its dependence on the depth in the $Z$ direction. As is plotted in Fig. 4(b), $J_{t}$ shows typical behavior of the Friedel oscillation, ${ }^{25}$ which maximizes at the top surface and decays to zero when moving away from the top surface. The gapless surface states on the side surfaces are conducting but they have vanishing net current density and hence do not contribute to the Hall conductance.

\section{MULTITERMINAL DEVICE TRANSPORT CALCULATION}

After establishing the existence of the chiral surface edge states, we can calculate the Hall conductance numerically using the Landauer-Büttiker formalism. ${ }^{26-28}$ The setup of the device is illustrated in Fig. 5(a): Four identical 2D metallic leads $(\mu=1,2,3,4)$ are attached to the top square surface of a semi-infinite 3D topological insulator, acting as the measurement electrodes. The Zeeman field is normal to the top surface. The multiterminal conductance can be deduced from the transmission coefficient $T_{p q}$ from the terminal $p$ to terminal $q ; T_{p q}=\operatorname{Tr}\left[\Gamma_{p} G^{r} \Gamma_{q} G^{a}\right]$ where $\Gamma_{p}$ is determined by the self-energy at the terminal $p \cdot{ }^{27}$ The calculated transmission coefficients as a function of Fermi energy $E_{f 1}$ in the $3 \mathrm{D}$ bulk
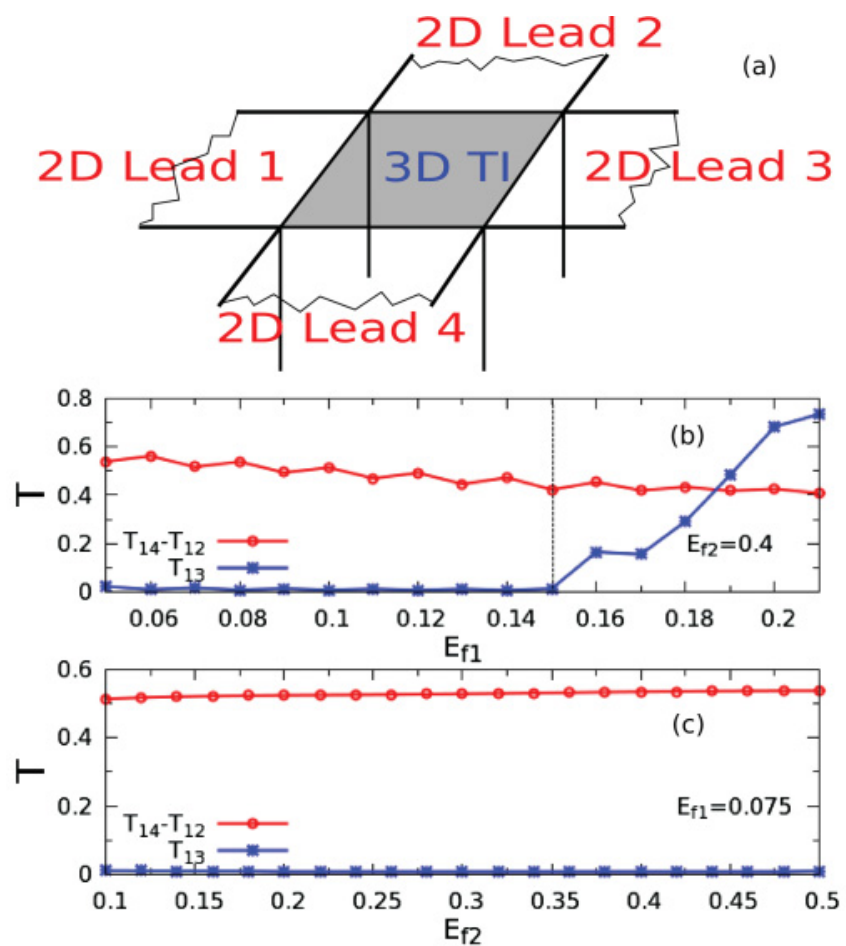

FIG. 5. (Color online) (a) Schematic illustration of the 3D device with $2 \mathrm{D}$ semi-infinite metallic leads; the sample is semi-infinite in the $Z$ direction, the top surface size is $30 \times 30$; (b) transmission coefficients of the four-terminal device, $E_{f 2}$ is fixed, the dashed line indicates the gap position; (c) $E_{f 1}$ is fixed. $\Delta_{z}=0.15, M=0.4$. and $E_{f 2}$ in the terminals are plotted in Figs. 5(c) and 5(d). When $E_{f 1}$ is located in the Zeeman gap of the surface states, the transmission coefficients show the "half chirality" (i.e., $T_{p q}-T_{q p} \approx \pm 1 / 2$ between the two neighboring terminals $p$ and $q$, and $T_{p q}=0$ between the non-neighboring terminals). This is different from the chirality shown in the usual quantum Hall systems, where $T_{p q}=n$ and $T_{q p}=0$ between the two neighboring terminals, where $n$ is an integer. It is also important to note that the transmission coefficient between the two neighboring terminals is always nonvanishing due to the presence of the gapless surface states in the side surfaces, and it is the difference between the clockwise and anticlockwise conducting channel that shows the "half quantization".

The above calculation shows that the Hall conductance is "half quantized" in the top surface. As is shown in Fig. 1, there are two such gapped surface Dirac cones on the top and bottom surfaces of the 3D TI. So when TI sample is thin enough, the Hall conductance on the two surfaces shall be measured simultaneously and unite to $e^{2} / h$. To check this picture, we can do a direct calculation with a TI sample that is of finite thickness in the $Z$ direction. Similarly, the four electrodes are only attached to the top surface and the Zeeman field is in the $Z$ direction. In this way, we can observe a transition from measuring "quantized" to "half-quantized" Hall conductance when increasing the sample thickness in the $Z$ direction. As is shown in Fig. 6 for a few sample sizes, in the thin film limit,
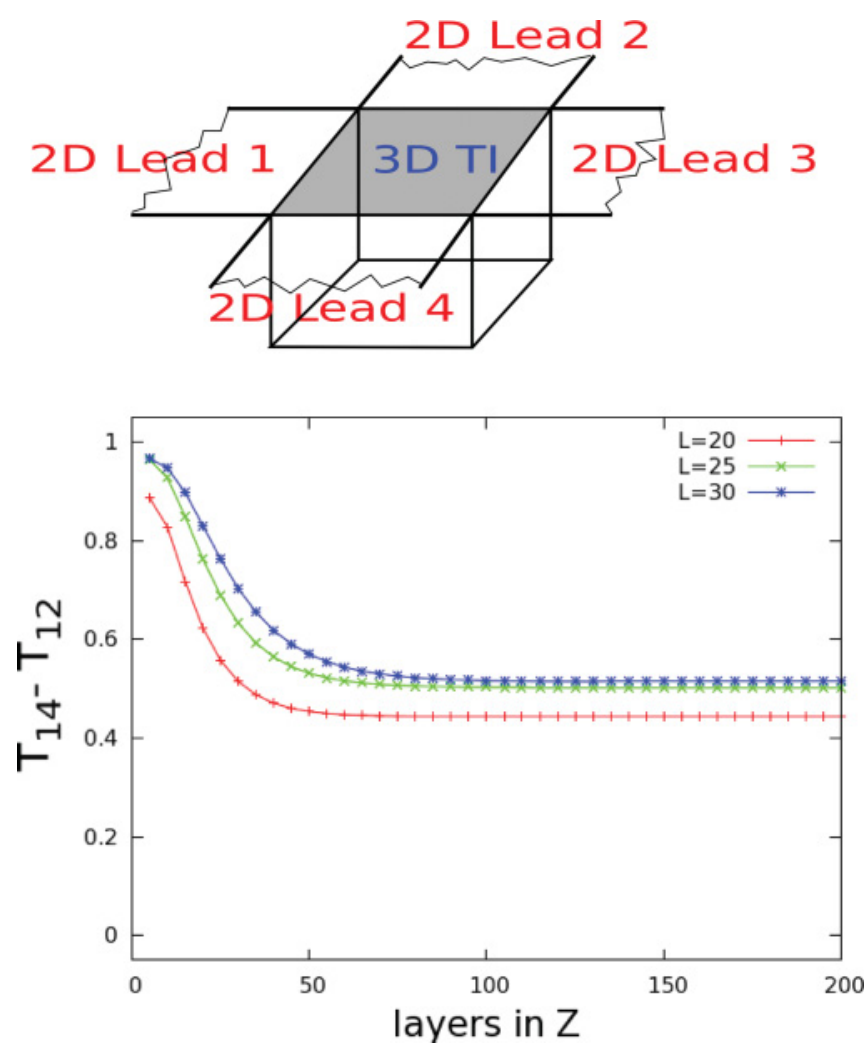

FIG. 6. (Color online) (Upper) Schematic illustration of the 3D device with $2 \mathrm{D}$ semi-infinite metallic leads; the sample has finite thickness in the $Z$ direction and the top surface size is $L \times L$. (lower) Transmission coefficients $T_{14}-T_{12}$ of the four-terminal device as a function of the sample thickness in $Z . \Delta_{z}=0.15, M=0.4, E_{f 1}=$ $0.001, E_{f 2}=0.04$. 
$T_{14}-T_{12}$ reaches the quantized value 1 , which implies that the quantized anomalous Hall effect exists in the thin TIs where spin-splitting terms are present. When we increase the layers in the $Z$ direction, $T_{14}-T_{12}$ gradually reduces and finally converges to a value close to 0.5 , which is consistent with the result where the sample is semi-infinite in the $Z$ direction.

\section{SUMMARY AND DISCUSSION}

A straightforward way to measure the "half-quantized" Hall conductance in the four-terminal setup is to apply a voltage between terminals 1 and $3\left(V_{13}\right)$, and measure the current between terminals 2 and $4\left(I_{24}\right)$. It is easy to show that the crossconductance $\sigma_{24,13} \equiv I_{24} / V_{13}=\left(e^{2} / 2 h\right)\left(T_{12}-T_{21}\right)$, yielding $e^{2} / 4 h$ for the half quantization. The measurement using the usual six-terminal Hall bar configuration could be more tricky due to the presence of the metallic side facets, which give rise to the finite longitudinal conductance $\sigma_{L}$. In the limit of the thick sample with $\sigma_{L} \gg e^{2} / h$, the Hall conductance $\sigma_{H}$ approaches $\left(4 e^{2} / h\right)\left(T_{p q}-T_{q p}\right)$ (if we assume $T_{p q}-T_{q p}$ is the same between all neighboring leads), which yields $2 e^{2} / h$ for the half quantization. It can be compared with the case of the quantum Hall effect where $\sigma_{L}$ vanishes when $\sigma_{H}$ shows quantization.

To summarize, we find chiral surface edge states in 3D topological insulators where a uniform spin-splitting Zeeman field is present. Effectively, one can consider that the original chiral edge mode carrying one conductance quantum $e^{2} / h$ is split into two halves concentrating at the interfaces of the insulating and metallic domains. Its origin is quite different from those in integer quantum Hall and quantum spin Hall effect, in which there exist well-defined chiral edge states. Here, many conducting channels, each contributing a fraction of the chirality, give rise to an effective surface edge state, as is indicated by the spatial distribution of the chiral edge current.

We have shown how the half quantization manifests itself in the multiterminal transport properties. Moreover, the existence of the effective surface edge states may be directly observable. For example, because the spin polarization of Dirac fermions in the surface states is proportional to the charge current, the spatial oscillatory pattern of the chiral edge current associating with the effective edge state could be directly probed by the spatially resolved Kerr rotation technique. ${ }^{29}$

\section{ACKNOWLEDGMENTS}

The authors thank Z. Fang, Q. F. Sun, W. Yao, and F. C. Zhang for helpful discussions. This work was supported by the Research Grant Council of Hong Kong under Grants No. HKU 7037/08P and No. HKUST3/CRF/09.
${ }^{1}$ J. E. Moore, Nature (London) 464, 194 (2010).

${ }^{2}$ X. L. Qi and S. C. Zhang, Phys. Today 63, 33 (2010).

${ }^{3}$ Z. Hasan and C. L. Kane, Rev. Mod. Phys. 82, 3045 (2010).

${ }^{4}$ L. Fu, C. L. Kane, and E. J. Mele, Phys. Rev. Lett. 98, 106803 (2007).

${ }^{5}$ J. E. Moore and L. Balents, Phys. Rev. B 75, 121306 (2007).

${ }^{6}$ D. Hsieh, D. Qian, L. Wray, Y. Xia, Y. S. Hor, R. J. Cava, and M. Z. Hasan, Nature (London) 452, 970 (2008); D. Hsieh, Y. Xia, L. Wray, D. Qian, A. Pal, J. H. Dil, J. Osterwalder, F. Meier, G. Bihlmayer, C. L. Kane, Y. S. Hor, R. J. Cava, and M. Z. Hasan, Science 323, 919 (2009).

${ }^{7}$ H. J. Zhang, C. X. Liu, X. L. Qi, X. Dai, Z. Fang, and S. C. Zhang, Nature Phys. 5, 438 (2009).

${ }^{8}$ Y. Xia, D. Qian, D. Hsieh, L. Wray, A. Pal, H. Lin, A. Bansil, D. Grauer, Y. S. Hor, R. J. Cava, and M. Z. Hasan, Nature Phys. 5, 398 (2009).

${ }^{9}$ Y. L. Chen, J. G. Analytis, J. H. Chu, Z. K. Liu, S. K. Mo, X. L. Qi, H. J. Zhang, D. H. Lu, X. Dai, Z. Fang, S. C. Zhang, I. R. Fisher, Z. Hussain, and Z. X. Shen, Science 325, 178 (2009).

${ }^{10}$ T. Yokoyama, Y. Tanaka, and N. Nagaosa, Phys. Rev. B 81, 121401(R) (2010).

${ }^{11}$ I. Garate and M. Franz, Phys. Rev. Lett. 104, 146802 (2010).

${ }^{12}$ A. N. Redlich, Phys. Rev. D 29, 2366 (1984).

${ }^{13}$ R. Jackiw, Phys. Rev. D 29, 2375 (1984); A. M. J. Schakel, ibid. 43, 1428 (1991).
${ }^{14}$ X. L. Qi, T. L. Hughes, and S. C. Zhang, Phys. Rev. B 78, 195424 (2008).

${ }^{15}$ X. L. Qi, R. D. Li, J. D. Zang, and S. C. Zhang, Science 323, 1184 (2009).

${ }^{16}$ A. M. Essin, J. E. Moore, and D. Vanderbilt, Phys. Rev. Lett. 102, 146805 (2009).

${ }^{17}$ B. I. Halperin, Phys. Rev. B 25, 2185 (1982).

${ }^{18}$ A. H. MacDonald and P. Streda, Phys. Rev. B 29, 1616 (1984).

${ }^{19}$ W. Y. Shan, H. Z. Lu, and S. Q. Shen, New J. Phys. 12, 043048 (2010).

${ }^{20}$ D. H. Lee, Phys. Rev. Lett. 103, 196804 (2009).

${ }^{21}$ H. Z. Lu, W. Y. Shan, W. Yao, Q. Niu, and S. Q. Shen, Phys. Rev. B 81, 115407 (2010).

${ }^{22}$ Rui-Lin Chu, Wen-Yu Shan, Jie Lu, and Shun-Qing Shen, Phys. Rev. B 83, 075110 (2011).

${ }^{23}$ S. Murakami, New J. Phys. 9, 356 (2007).

${ }^{24}$ M. P. L. Sancho, J. M. L. Sancho, and J. Rubio, J. Phys. F 14, 1205 (1984).

${ }^{25}$ C. Kittel, Introduction to Solid State Physics, 7th ed. (John Wiley \& Sons, New York, 1996).

${ }^{26}$ M. Buttiker, Phys. Rev. Lett. 57, 1761 (1986).

${ }^{27}$ S. Datta, Electronic Transport in Mesoscopic Systems (Cambridge University Press, Cambridge, 1995).

${ }^{28}$ J. Li, L. Hu, and S. Q. Shen, Phys. Rev. B 71, 241305(R) (2005).

${ }^{29}$ Y. K. Kato, R. C. Myers, A. C. Gossard, and D. D. Awschalom, Science 306, 1910 (2004). 